\title{
Estructuración socio territorial del departamento de Arauca, 1950-2008
}

\author{
Socio territorial structuring of the Colombian Department \\ of Arauca, 1950 2008
}

Betty Esther Mendoza Padilla ${ }^{1}$

\section{Resumen}

En la estructuración socio territorial del actual departamento de Arauca se identificaron cuatro procesos predominantes desde 1950 hasta 2008. Primero, el poblamiento departamental en áreas rurales y cabeceras municipales que presenta dos comportamientos caracterizados por un crecimiento gradual entre 1951 y 1985 y un acelerado poblamiento entre 1985 y 2005 . Segundo, la posesión de tierras utilizada como mecanismo de dominio territorial mediante su concentración, lo que produce el conflicto de uso de suelos en los espacios rurales. Tercero, el conflicto armado en la década del sesenta aparece como un componente determinante en los cambios espaciales departamentales. Y cuarto, el descubrimiento del campo Caño Limón en 1983, que marca el momento en que el petróleo se erige como agente histórico modificador del espacio araucano y de integración del departamento a la nación. Para este trabajo se aplicó el método geohistórico por medio del análisis secuencial de acontecimientos, utilizando técnicas de investigación histórica.

Palabras clave: Conflicto armado, estructuración socio territorial, petróleo, poblamiento, posesión de tierras.

1 Magister en Geografía. Profesional especializado, Instituto Geográfico Agustín Codazzi IGAC. Bogotá D.C.- Colombia. bettyemendoza@gmail.com,bmendoza@igac.gov.co. 


\section{Abstract}

Nowadays, in socio-territorial structuring of the Arauca department, four predominant processes were identified from 1950 through 2008. First, the settlement in rural and municipal areas presents two behaviors characterized by a gradual growth between 1951 and 1985 and an increased rate of settlement between 1985 and 2005. Second, the ownership of land was used as a mechanism for territorial dominance which resulted in land concentration and conflicts of land use in rural areas. Third, the armed conflict in the 60's emerged as a decisive component in the spatial changes of the department. Finally, in 1983 when the Caño Limón oilfield was discovered, oil emerged as a historical agent modifying the space of Arauca and its integration into the nation. Geohistorical method was applied through the sequential analysis of events, using techniques of historical research.

Key words: Armed conflict, socio-territorial structuring, oil, ownership land, settlement. 


\section{Introducción}

El espacio geográfico es el resultado de transformaciones permanentes que se producen a través del tiempo mediante sistemas de relaciones que se establecen entre los elementos del escenario físico -arquitectura de los volúmenes rocosos, clima, vegetación, ecosistemas- y los grupos sociales. Así, los espacios se estructuran como efecto de las decisiones humanas tomadas a través del tiempo y según temporalidades históricas, respecto de las posibilidades reales que ofrece la naturaleza, y en función de la densidad de poblamiento, la organización social y económica, y el nivel de las técnicas productivas; en síntesis, de todo el denso tejido histórico que constituye una civilización (Dollfus, 1976).

Partiendo de esta idea, un territorio sufre sistemáticos cambios en sus características internas y en sus temporalidades de estructuración debido a la acción de fuerzas humanas sobre las estructuras físico-naturales que las soportan, y, en determinadas circunstancias, debido a la acción de fuerzas de la naturaleza en los procesos formativos, ya sea por su evolución natural, o bien por un evento catastrófico. En este sentido, el análisis de las estructuras de un espacio o territorio determinado, no solo permite conocer la distribución de los asentamientos, sino también sus interrelaciones espaciales, sus grados de especialización, sus potencialidades y las diferencias existentes entre ellas, y la delimitación de unidades internas, entre otros, además de ser una herramienta útil para señalar los elementos esenciales que determinan las líneas fundamentales de la política territorial que se aplica.

La configuración territorial o geográfica, que para Santos (1996) es el territorio, más el conjunto de los objetos existentes en él, tanto naturales como artificiales, es el resultado en el tiempo de procesos históricos, lo cual significa que no aparecen de un momento a otro ni que adquieren ciertas características en un momento dado. Para descubrir si un objeto es natural o artificial hay que comprender su historia, sostiene Santos. En el discernimiento de la configuración territorial, Santos (2000) introduce además el enfoque sistémico, cuando señala que la configuración territorial "está determinada por el conjunto formado por los sistemas naturales existentes en un país determinado o en un área dada y por los agregados que los hombres han sobrepuesto a esos sistemas naturales". La configuración territorial o configuración geográfica, tiene por ello, una existencia material propia, pero su existencia social, es decir, su existencia real, solamente le viene dada por el hecho histórico de las relaciones sociales. En este marco teórico se estudia la configuración socio territorial de una porción de la Orinoquia colombiana, el actual departamento de Arauca. Las temporalidades en que se dan los procesos -periodos históricos-, constituyen los fundamentos del análisis de la estructura de un espacio geográfico, dentro del marco de la geohistoria.

La contribución práctica que se pretende dar a la ciencia de la geohistoria con el trabajo de investigación sobre el departamento de Arauca, es analizar en cada 
nuevo proceso de reestructuración que tiene lugar en el espacio, las circunstancias históricas del escenario físico y de los aconteceres del accionar de las sociedades que lo habitan, para establecer si el rol de la misma configuración física del territorio -atributos propios del escenario natural- es el determinante principal en dicha reestructuración del espacio geográfico, o si es procedente prioritariamente del actuar de la sociedad local o externa al espacio de estudio en un momento de su realidad histórica. En el primer caso, no se trata de demostrar un determinismo geográfico, sino que se asume el escenario físico como el soporte de los diferentes momentos históricos, que influye en las sociedades pero que no las determina, puesto que las mismas sociedades con su tecnología pueden llegar a cambiar la propia geografía en la que se asientan (Braudel, 1950). El interrogante fundamental lo expresa Grataloup (1996): ¿es el espacio en un momento dado de su temporalidad factor predominante de historia?, o bien, ¿es el espacio en un momento dado de su temporalidad sujeto y efecto de hechos históricos predominantes de las sociedades internas o externas? Es decir, como afirma Tovar (1996), que el espacio físico adquiere una dinámica, complejidad, heterogeneidad y especificidad que lo define como factor predominante de cambios y estructuras en su historia, o en el caso contrario, el espacio es el sujeto de los aconteceres temporales.

Si mediante el análisis de las dimensiones cualitativas, al identificar y explicar los hechos históricos que originaron y deter- minaron cambios en la estructura espacial de Arauca, se concreta que el espacio natural, en alguna de sus características del momento histórico, desempeña el rol predominante que inicia y desarrolla un nuevo proceso de configuración espacial, y que define permanencias históricas en la dinámica social, entonces se propone identificar la dimensión natural como el factor principal de historia, o de momentos geohistóricos. Por ejemplo, los yacimientos de petróleo en el subsuelo, cuyo descubrimiento y explotación generaron dinámicas y nuevas configuraciones espaciales. Además, se tiene en cuenta la localización y distribución en el territorio de formas económico-productivas y sociales específicas, junto con la aparición de concentraciones humanas. $\mathrm{Si}$, por el contrario, se razona que los hechos históricos sucedidos en el espacio de Arauca, o fuera, pero con influencia sobre este, son los determinantes principales de los procesos de reestructuración territorial, se propone identificar el espacio como efecto o resultado de hechos históricos que le fijaron procesos que estructuraron la actual configuración geográfica. Por ejemplo, las distintas delimitaciones y categorías político-administrativas instauradas desde la capital de la República, o la construcción, localización y distribución de infraestructuras regionales de diverso tipo.

En relación con el método geohistórico, Hurtado (1994) señala que por lo general, las estructuras del espacio geográfico son producto de dinámicas sociales, sus formas y contenidos cambian con la historia, de ahí la importancia del uso de este método, e indica también, que el 
espacio geográfico no se puede observar de manera homogénea, ni estática y recomienda definir los parámetros cronológicos y espaciales que interesan en los estudios.

Teniendo en cuenta el soporte conceptual descrito, el objetivo es precisar y examinar los factores predominantes, efectos y lógicas que dieron lugar en el periodo de 1950-2008, a progresivas producciones de espacio en la configuración territorial del actual departamento de Arauca, en relación con la ocupación humana, las formas y prácticas sociales, el desarrollo de sus sistemas económico-productivos y la infraestructura de comunicaciones.

Como hipótesis, se parte del supuesto que las distintas relaciones socioeconómicas de los grupos humanos que han ocupado y que con mayor incidencia han estructurado en temporalidades identificadas los diferentes espacios que conforman el actual departamento de Arauca, son el resultado de hechos predominantes y sobresalientes, por una parte, inherentes a propiedades del mismo espacio (escenario físico), como por ejemplo, los recursos hidrocarburíferos, y por otra, de circunstancias surgidas y maduradas fuera del ámbito departamental, como la implementación de políticas de incentivos a la producción agropecuaria, o de protección de territorios indígenas.

A fin de alcanzar los objetivos propuestos, se aplica el método geohistórico por medio del análisis secuencial de acontecimientos, utilizando técnicas de investigación histórica en los distintos análisis e inferencias mediante análisis multitemporales y holísticos. El método geohistórico tiene la ventaja de considerar al "espacio" y al "tiempo" como concretos.

\section{Transformación de la estructura poblacional}

La estructura del actual departamento de Arauca se construyó progresiva y simultáneamente con el territorio nacional, a partir de procesos, de momentos históricos, de intereses económicos y del carácter cultural de sus habitantes.

Uno de los dinamizantes espaciales en la configuración de un territorio es la estructura demográfica, cuyas características, interacciones y dinámicas determinan en última instancia, los ritmos, temporalidades y tasas de formación y transformación de sus sociedades y, por medio de estas, de los entornos naturales. Se podría afirmar en tal sentido que sin la intervención de fuerzas humanas en los espacios, se hablaría de naturaleza prístina que a estas alturas de la historia de la humanidad es muy poco probable encontrarla, particularmente en la unidad espacial objeto del presente estudio, como es el departamento de Arauca.

Teniendo en cuenta lo anterior, la demografía departamental entre el censo de 1951 y 2005, según datos del Departamento Administrativo Nacional de Estadística, se caracteriza por el acelerado crecimiento demográfico que a nivel nacional incrementó su población en 3,7 veces, de $11^{\prime} 548.178$ a $42^{\prime} 888.592$ ha- 
bitantes respectivamente, superándose en Arauca este incremento, muy superior, de 13.221 a 232.118 habitantes, 26,4 veces al valor nacional.

En términos generales, se identificaron dos periodos; el primero, entre 1951 y 1985, y el segundo, entre 1985 y 2005 , notoriamente diferenciados por su composición poblacional, teniendo como factores, la movilidad interna, la violencia política y circunstancias económicas tales como la aceleración tecnológica generada por presiones externas en la imposición de un modelo de crecimiento económico, basado en la sobreexplotación de los recursos naturales y en el desplazamiento de la mano de obra.

\subsection{Crecimiento gradual de la población de Arauca, 1951-1985}

En 1951, el actual departamento de Arauca contaba con un total de 13.221 habitantes (DANE, Censo 1951, ) de los cuales un $28 \%$ correspondía a la población urbana y el resto (72\%) estaba distribuido en el área rural. Para 1985, el total de habitantes del departamento era de 70.085, de los cuales el porcentaje de población urbana correspondía al 58 $\%$ y la población rural al $42 \%$. Durante este periodo de 34 años, la población total aumentó en 5,3 veces, a un ritmo de incremento anual de población de 1.672 habitantes, evidenciándose un crecimiento gradual de población en los centros urbanos en los periodos intercensales de 1964 y 1973, como se puede advertir en la Figura 1.
Por otra parte, en 1951 la mayor proporción de la población (66 \%) se concentró en el municipio de Arauca, capital del actual departamento, seguido del municipio de Tame (27\%), teniendo el municipio de Arauquita (8\%) la menor cantidad de población del departamento. Para entonces no se habían creado los municipios de Saravena, Fortul, Cravo Norte y Puerto Rondón.

En 1985, la población total departamental fue de 70.085 habitantes, lo cual significa 1,5 veces más de población respecto a 1973, lo que representa un incremento anual poblacional de 1.956 habitantes, valor superior al incremento anual (1.517) observado antes de este año. Entre 1973 y 1985 se observa el quiebre de la composición poblacional departamental campo-ciudad, pasando a partir de 1985 a predominar la población asentada en los centros urbanos, como se observa en la Figura 1. Dicha tendencia en la relación de la población campociudad se aceleró a partir de 1950 con la implementación de políticas de expansión de la frontera agrícola, materializada en ondas de colonización de poblaciones migratorias de las partes altas del departamento, que ocuparon espacios de llanuras del mismo.

\subsection{Aceleración del crecimiento y concentración de población en Arauca, 1985-2008}

El descubrimiento del campo petrolero Caño Limón en el municipio de Arauca en 1983, propició un significativo incremento de la cantidad de habitantes 
Figura 1. Población urbana y rural de Arauca, 1951-2005

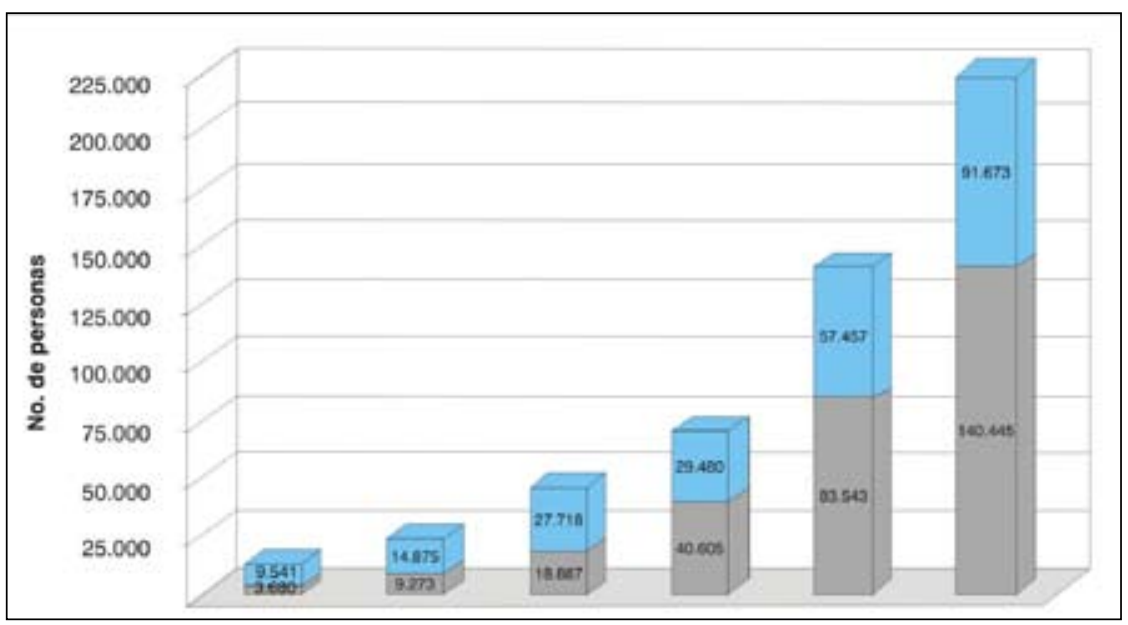

Fuente: DANE, censo 1951-2005

urbanos, particularmente concentrada en este municipio a partir de 1985 , como se puede advertir en la Figura 1. Es así como para 1993 la población total del departamento era de aproximadamente 141.000 habitantes, duplicando prácticamente la población de 1985 , con un incremento anual de 8.864 habitantes, muy superior a los presentados antes de 1993. Esta tendencia del crecimiento de la población departamental se mantuvo hasta 2005, cuando el censo arrojó un total de 232.118 habitantes, aunque se percibe en la cifra de 7.593 habitantes/año, un ligero decrecimiento de la velocidad de asentamiento anual en el periodo 1993-2005 comparado con los 8.864 del periodo 1985-1993 (Tabla 1 y Figura. 2).

Tabla 1. Población urbana y rural municipal de Arauca, 1985-2005

\begin{tabular}{|c|c|c|c|c|c|c|c|c|c|}
\hline Municipios & \multicolumn{3}{|c|}{ Población Urbana } & \multicolumn{3}{c|}{ Población Rural } & \multicolumn{3}{c|}{ Población Total } \\
\hline Años & $\mathbf{1 9 8 5}$ & $\mathbf{1 9 9 3}$ & $\mathbf{2 0 0 5}$ & $\mathbf{1 9 8 5}$ & $\mathbf{1 9 9 3}$ & $\mathbf{2 0 0 5}$ & $\mathbf{1 9 8 5}$ & $\mathbf{1 9 9 3}$ & $\mathbf{2 0 0 5}$ \\
\hline Arauca & 15.813 & 38.703 & 62.634 & 5.466 & 815 & 12.923 & $\mathbf{2 1 . 2 7 9}$ & 39.518 & 75.557 \\
\hline Arauquita & 3.283 & 5.144 & 15.078 & 7.021 & 18.238 & 21.667 & 10.304 & 23.382 & 36.745 \\
\hline Saravena & 11.128 & 19.132 & $\mathbf{2 8 . 5 4 4}$ & 8.180 & 6.093 & 14.222 & 19.308 & 25.225 & 42.766 \\
\hline Fortul & & 2.344 & 10.009 & & 10.466 & 11.842 & & 12.810 & 21.851 \\
\hline Cravo Norte & 1.120 & 3.239 & 2.391 & 1.557 & 979 & 1.270 & 2.677 & 4.218 & 3.661 \\
\hline Puerto Rondón & 708 & 2.099 & 2.655 & 799 & 3.578 & 1.307 & 1.507 & 5.677 & 3.962 \\
\hline Tame & 8.553 & 12.882 & 19.134 & 6.457 & 17.288 & 28.442 & 15.010 & 30.170 & 47.576 \\
\hline Total & 40.605 & 83.543 & 140.445 & 29.480 & 57.457 & 91.673 & 70.085 & 141.000 & 232.118 \\
\hline
\end{tabular}

Fuente: Censos Nacionales de Población y Vivienda. Departamento Administrativo Nacional de Estadística 
Figura 2. Población urbano- rural y densidad demográfica de Arauca, 2005.

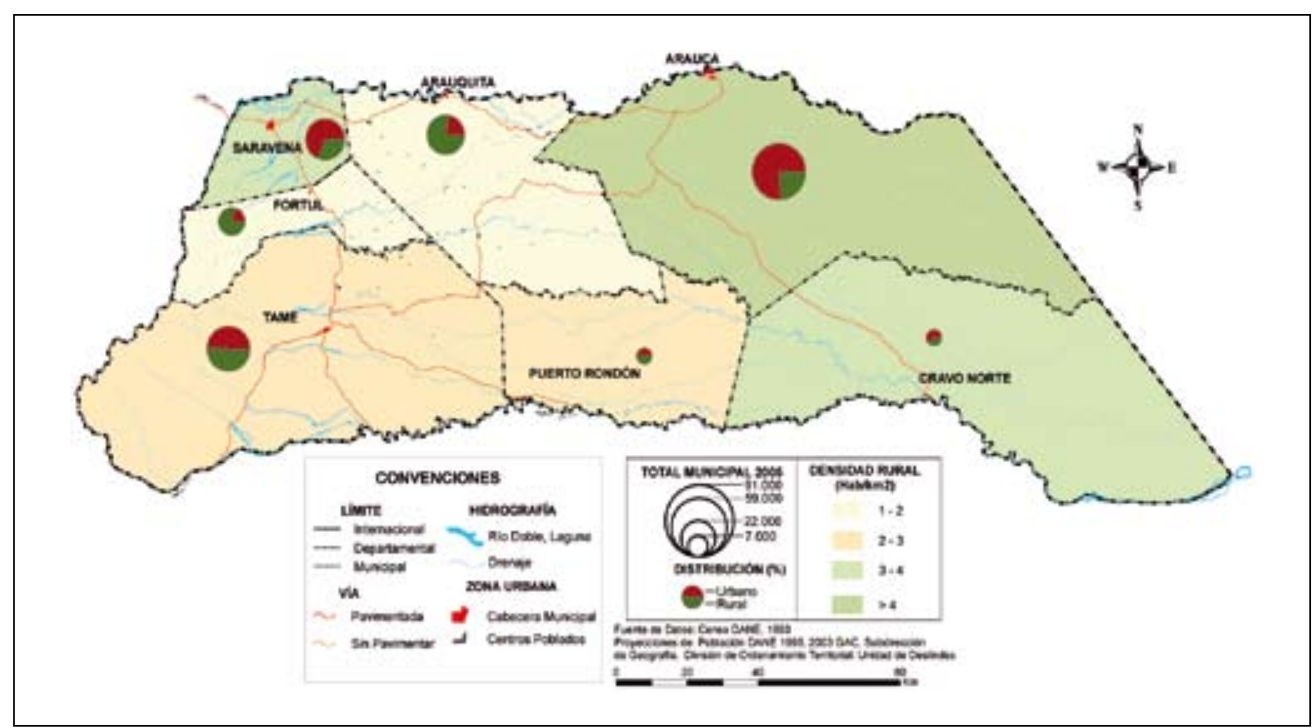

Fuente: elaboración propia, con base en Sistema de Información Geográfica departamento de Arauca, 2006

Así mismo, se advierte que en los años 1985, 1993 y 2005, la población urbana se mantuvo en términos generales en similares niveles porcentuales respecto de sus totales, variando de $58 \%$ a $61 \%$, entre 1985 y 2005 , lo cual significa que la relación de población urbano-rural permaneció relativamente estable.

Este fenómeno de poblamiento en Arauca es ejemplificado por Avellaneda (2004), quien señala que los caseríos, como Arauquita, crecieron aceleradamente cuando las petroleras en 1984-1986 establecieron sus campamentos para iniciar operaciones y construir el oleoducto en el tramo Caño Limón-Río Zulia, por la empresa alemana Mannesman.
Otro factor diferencial de la población es la migración, en la que se dan dos fenómenos: la inmigración o entrada de personas a un territorio y emigración o salida de residentes de una zona. Las emigraciones del departamento son causadas principalmente por el conflicto armado. Por su parte, las inmigraciones presentaron un incremento notable a partir del auge petrolero. Según el censo de 1993, los departamentos de Santander, Norte de Santander, Boyacá, Casanare y Meta son los que más aportan población migratoria al departamento con $10 \%, 9 \%, 4 \%, 4$ $\%$ y $2 \%$, respectivamente, el resto de departamentos del país le aportan un 13\% del total de la población de Arauca. 
Las migraciones forzadas o desplazamientos forzados se dan a raíz de la violencia originada por grupos armados al margen de la ley: autodefensas, guerrillas y delincuencia común. Por cada 1.000 habitantes en el departamento, 10 han sufrido desplazamientos forzados hacia otras zonas del país o hacia las áreas urbanas del mismo departamento. En el periodo 2002 al 2005, se han visto afectadas 20.494 personas por este fenómeno (Acción Social, 2005). Arauca ocupa el puesto número 23 a nivel nacional en recepción de población desplazada y el 19 en expulsión de población. Tame, Arauca y Saravena son los municipios que más población expulsan y a su vez los que más población reciben (Red de Solidaridad Social, 2005).

\subsection{Flujos migratorios y su composición}

Al mismo tiempo que la población se incrementaba en los núcleos urbanos del actual departamento de Arauca, durante el periodo 1951-1985 se registraba la disminución de población de las áreas rurales. La migración dentro del departamento (campo-ciudad), se debió, entre otros factores, a los procesos de colonización latifundista, los cuales desplazaron asentamientos locales dispersos, principalmente distribuidos en las unidades geográficas sabaneras, y posteriormente por la intensificación de las actividades de exploración petrolífera, a inicios de la década del 70. Con ello se originó la llegada de grupos de población de otros departamentos, principalmente de las zonas andinas, población que si bien desarrollaba sus labores en los campos y pozos petroleros de las áreas rurales, concentraba su cotidianidad en las áreas urbanas que ofrecían servicios públicos y sociales para el desarrollo de los habitantes. Igualmente llegaron los grupos al margen de la ley.

\section{Posesión de tierras como mecanismo de dominio territorial}

La concentración de tierras que se presenta en el departamento de Arauca en la actualidad, tiene su origen en los primeros procesos de colonización sobre tierras vírgenes del país que se inicia a partir de la primera década del siglo XIX. En general, el patrón de colonización que se desarrolló estuvo basado en el modelo conocido como de "endeude" y de manera muy esquemática consistía en que al campesino pobre, por lo común migrante por necesidad o violencia, se le ofrecía un préstamo para que iniciara una "mejora" en el terreno baldío que se le adjudicó, y una vez que había transformado un área de selva e iniciado una pequeña producción agrícola, se le cobraba la deuda, la cual no tenía con qué pagar puesto que la producción era de pan coger, que no genera dividendos de ahorro; en consecuencia, generalmente debía pagar con la tierra, que pasaba al comerciante, el cual podía acumularla o, a su vez, venderla a un latifundista (Márquez, 2001). El proceso continúa, puesto que el colono ocupa una nueva porción de tierra, que transforma y entrega de nuevo en pago, esporádicamente logra conservarla en condiciones más o menos 
satisfactorias. En concreto, se trata de la explotación de campesinos pobres por parte de comerciantes y terratenientes, los que en definitiva se beneficiaron del proceso.

La incidencia del proceso de numerosos migrantes determina la transformación de las selvas en potreros que tienden a quedar en manos de unos pocos propietarios. Este patrón parecía haber agotado sus posibilidades hacia 1980, cuando las tierras con vocación agrícola y ganadera, relativamente cercanas a los centros de mercadeo, se estaban agotando; no obstante, la economía del narcotráfico introdujo nuevos incentivos a la colonización, esta vez basada en el tráfico de los cultivos ilícitos. El esquema sigue siendo similar, con la posibilidad de ganancias extraordinarias y también de mayores endeudamientos. La concentración de la propiedad se ha acelerado, dada la costumbre de reunir tierras como parte de una estrategia de poder económico y político y de control territorial. Merced a ello, y a circunstancias de violencia que expulsan de sus propiedades a quienes no estén en capacidad de defenderlas o pagar, la concentración de las tierras es hoy mayor que nunca en la historia del país, en especial en zonas de colonización reciente, como es el caso de Arauca (Márquez, 2001).

\section{Concentración de tierras y conflictos de uso del suelo en la sabana de Arauca}

La característica fundamental del departamento es la escasa presencia de una estructura de minifundio, en consecuencia, una apropiación extensiva de la tierra, por lo tanto predomina el latifundio en la sabana, el que comparte con una estructura de mediana propiedad en el piedemonte araucano. La gran extensión, con hato ganadero, es sinónimo de "tradición llanera" (Centro de Investigaciones para el Desarrollo, Universidad Nacional de Colombia, 2005). La actividad agropecuaria es la más representativa del departamento, dispone de $23.818 \mathrm{~km}^{2}$ y de una estructura de reparto de medianas a grandes extensiones (Instituto Geográfico Agustín Codazzi [IGAC], 2005).

De acuerdo con reportes del Instituto Geográfico Agustín Codazzi (2005), en el departamento de Arauca existen pocos predios y propietarios respecto del tamaño de la tierra existente, los que se distribuyen entre particulares y agencias del Estado. Los predios registrados en el 2005 fueron 15.455 y 17.561 propietarios, con una superficie muy cercana al total del área del departamento (Tabla 2); esto quiere decir que allí estaban el 0,46 $\%$ de los propietarios rurales del país, con el $0,56 \%$ de los predios y una posesión del 3,07 \% de la tierra registrada catastralmente.

De acuerdo con el área nacional, el departamento de Arauca posee el 2,09\% de la superficie del país y si tuviera una estructura de propiedad similar a la del 
Tabla 2. Distribución rural (\%) por rangos de superficie departamento de Arauca, 2005

\begin{tabular}{|c|c|c|c|}
\hline Tamaño & Predios & Propietarios & Superficie \\
\hline Menos de 1 ha & 3,04 & 3,11 & 0,005 \\
\hline De 1 a 3 has & 2,59 & 2,42 & 0,029 \\
\hline De 3 a 5 has & 4,16 & 4,04 & 0,098 \\
\hline De 5 a 10 has & 9,48 & 9,15 & 0,420 \\
\hline De 10 a 15 has & 9,10 & 9,12 & 0,677 \\
\hline De 15 a 20 has & 7,45 & 7,35 & 0,786 \\
\hline De 20 a 50 has & 32,16 & 31,83 & 6,522 \\
\hline De 50 a 100 has & 16,94 & 17,21 & 7,071 \\
\hline De 100 a 200 has & 6,56 & 6,62 & 5,143 \\
\hline De 200 a 500 has & 4,25 & 4,60 & 8,165 \\
\hline De 500 a 1000 has & 2,34 & 2,42 & 10,418 \\
\hline De 1000 a 2000 has & 1,13 & 1,29 & 9,199 \\
\hline Más de 2000 has & 0,80 & 0,91 & 51,198 \\
\hline Total & $\mathbf{1 5 . 4 5 5}$ predios & $\mathbf{1 7 . 5 6 1}$ propietarios & $\mathbf{2 . 5 2 2 . 7 6 1 ~ h a}$ \\
\hline
\end{tabular}

Fuente: Instituto Geográfico Agustín Codazzi. Cálculos CID-UN (2005).

resto, debería tener, al menos, tres veces más de predios y de propietarios. Esta estructura de mediana y gran propiedad no está acompañada de una actividad agropecuaria eficiente, razón que, aunada a su relativo aislamiento del resto del país y a la inseguridad generada por el conflicto armado, que afecta también a varias regiones del país, originó un mercado de tierras poco activo con precios relativamente bajos (Centro de Investigaciones para el Desarrollo, 2005).

La estructura de la propiedad rural araucana revela la presencia de cuatro gran- des grupos de propietarios. El latifundio, de predios de más de 2.000 ha, donde 159 propietarios, públicos y privados, poseen el 51,2 \% del área registrada catastralmente, con un promedio de 8.123 ha por propietarios, al compararse con la estructura nacional (Tabla 3) representan al 6,5\% con el 2,9\% de esta tierra. Esto quiere decir que hay latifundios aun más grandes en otras regiones del país, sin embargo, existe un predio de 45.000 ha en Fortul, otro de 17.000 en Saravena y el promedio de la gran propiedad en Tame es de 22.000 ha.

Tabla 3. Distribución predial rural departamental y nacional por rangos de superficie, en \%

\begin{tabular}{|c|c|c|c|c|}
\hline \multirow{2}{*}{ Tamaño } & \multicolumn{2}{|c|}{ Propietarios } & \multicolumn{2}{c|}{ Superficie } \\
\cline { 2 - 5 } & Arauca & Nacional & Arauca & Nacional \\
\hline Menos de 20 ha & 35,2 & 86,6 & 2,0 & 8,8 \\
\hline De 20 a 100 ha & 49,0 & 10,4 & 13,6 & 14,6 \\
\hline De 100 a 2000 ha & 14,9 & 2,9 & 33,2 & 23,1 \\
\hline Más de 2000 ha & 0,9 & 0,06 & 51,2 & 53,5 \\
\hline
\end{tabular}

Fuente: Instituto Geográfico Agustín Codazzi. Cálculos CID-UN (2005). 
Esta particularidad se comprende mejor al contrastarla con el grupo de minifundistas, en que el $35 \%$ de propietarios poseen solamente el $2 \%$ de la tierra, con un promedio de 8,3 ha por predio, muy superior al valor nacional de 2,2 ha, es decir, que hasta los pequeños propietarios disponen de más tierra que sus pares en otras regiones.

La estructura predial descrita debería permitir un uso racional de las medianas y grandes propiedades, sobre todo cuando los diversos estudios sobre uso potencial revelan aptitudes diferentes a las actualmente usadas. El proceso de colonización terminó tumbando bosques, inicialmente para desarrollar algún cultivo agrícola y luego convertirlos en praderas para la ganadería. En medio siglo de colonización se estima una deforestación de 862.000 ha, dejando un bosque nativo de apenas 220.000 ha. Esa deforestación no implicó el desarrollo organizado de la actividad forestal ni la conservación de zonas de reserva (Centro de Investigaciones para el Desarrollo, 2005).

Además de la gran concentración de la propiedad rural en Arauca, confirmada en la curva de Lorentz de la figura No. 3 , cuyo índice de Gini estuvo muy por encima de 0,75 para el año 2005, existía el conflicto entre el uso real y el potencial de la tierra, dado que para la agricultura se utilizaban según CORPOICA (1997), alrededor de 40.000 ha, mientras existía una vocación de uso estimada en 500.178 ha, es decir, se estaba utilizando alrededor del $8 \%$ de la tierra apta. Con la ganadería sucedía todo lo contrario, a ella se destinaban 1,8 millones de hectáreas, mientras la vocación estimada era de apenas 1,5 millones de ha, es decir, había 300.000 ha de más en uso. La gran actividad sacrificada en este periodo fue

Figura 3. Curva de Lorentz: Grado de concentración de la propiedad rural, 2005.

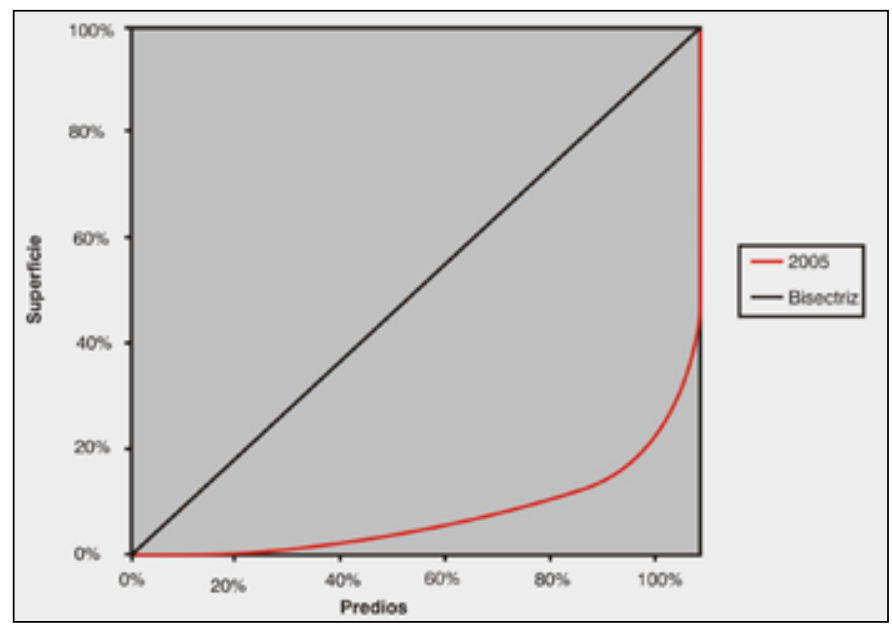

Fuente: elaboración propia, con base en datos del Instituto Geográfico Agustín Codazzi. Cálculos CID-UN, (2005). 
la forestal, para la cual se estimaba una potencialidad de 1,8 millones de ha, es decir el $76 \%$ del territorio araucano frente a 220.000 ha que tiene actualmente en bosques (Centro de Investigaciones para el Desarrollo, 2005).

\section{El conflicto armado como factor de cambios espaciales}

Desde el enfoque conceptual que sustenta el presente estudio, el conflicto armado y las derivaciones de carácter conflictivo que en este se apoyaron, se consideran como factores determinantes de cambios en la evolución de la estructura de los espacios geográficos de Arauca. Con la aparición formal de los grupos armados insurgentes en 1964, y posteriormente de los cultivos ilícitos y demás problemáticas correlacionadas, Colombia inicia un periodo en su historia contemporánea que marca hasta nuestros días la vida de la nacionalidad en varias de sus dimensiones, colocándolos en el escenario político como nuevos actores, afectando los comportamientos de la vida nacional en diferentes ámbitos, situación que afecta igualmente al departamento.

Las condiciones de lejanía de la Orinoquia respecto de la región andina central, la de mayor desarrollo socioeconómico de Colombia, sumado a la escasa población, gran extensión superficial y características naturales, motivaron la presencia en la década de los sesenta del siglo XX de los primeros grupos guerrilleros al margen de la ley, desarrollándose con fuerza y mayor permanencia como con- flicto armado, seguido y paralelo con la ocupación de importantes extensiones de cultivos de hoja coca, su producción y comercialización.

A partir de los años setenta se incrementa la penetración de grupos armados al margen de la ley: Ejército de Liberación Nacional (ELN), Fuerzas Armadas Revolucionarias de Colombia (FARC) y las Autodefensas Unidas de Colombia (AUC). Con el descubrimiento del campo petrolero de Caño Limón en 1983, además de las acciones extorsivas, los grupos armados ilegales utilizarían el recurso petrolero y su infraestructura como elementos coercitivos contra el Estado, con lo cual sus acciones cobrarían mayor valor como factor estructurante territorial, dada la importancia económica del campo petrolero para la región y el país.

En el transcurso de la década de los setenta hasta principios de los años ochenta, el ELN logró consolidarse en el departamento de Arauca, planteándose un nuevo modelo de organización, identificando como elementos claves de sus acciones las zonas petroleras, las vías de comunicación importantes entre ciudades, caracterizando detalladamente cada área y dándosele el carácter de independiente, lo cual siguió marcando el accionar del ELN en Arauca y manteniendo su posición territorial en los años siguientes. Las acciones de este grupo en Arauca, inicialmente motivadas por el desarrollo de luchas agrarias, pronto estarían relacionadas con la explotación petrolera debido a su experiencia armada en zonas del Magdalena Medio, especial- 
mente en los municipios de Cimitarra y Barrancabermeja. Las FARC hicieron su aparición en Arauca a finales de los años setenta con un radio de acción hacia el Sarare y la Sierra Nevada del Cocuy. La organización guerrillera abrió un nuevo núcleo de expansión con influencia en el extremo norte de la cordillera Oriental y en la frontera con Venezuela, que en los años siguientes le sirvió de base para registrar un crecimiento sin precedentes a nivel nacional y particularmente en el oriente del país (Vicepresidencia de la República, 2003).

El conflicto armado tuvo como principales mecanismos de acción la emboscada y los ataques a las infraestructuras del oleoducto Caño Limón y empresas contratistas, afectando todos los municipios del departamento, en especial Tame, Saravena y Arauquita, además extiende su presencia a municipios limítrofes del departamento de Arauca como Hato Corozal y Paz de Ariporo en Casanare (Vicepresidencia de la República, 2003).

A comienzos de los años 90, la ocupación territorial de las FARC se extiende y cubre buena parte de los municipios del departamento de Arauca afectando todo el piedemonte limítrofe con Casanare (Vicepresidencia de la República, 2003). El ELN, por su parte, sostiene una intensa actividad en casi todo el departamento, pero en particular en Tame. Durante este tiempo la actividad armada se caracteriza por la destrucción de infraestructuras. El incremento de homicidios, la presión de la guerrilla sobre el oleoducto y las compañías petroleras, llevaron al gobierno a la creación de la Zona de Rehabilitación en el departamento, con un importante apoyo económico del gobierno de los Estados Unidos de cerca de 98 millones de dólares (Vicepresidencia de la República, 2003).

Las denominadas Autodefensas hicieron su aparición en 1993 en el departamento de Arauca, especialmente en el municipio de Saravena. La presencia inicial estuvo ligada al apoyo de los grupos asentados en el vecino departamento de Casanare, desde donde se realizaron en los años 2000 y 2001 las primeras incursiones hasta que éstas lograron asentarse en las cabeceras municipales de Tame, Puerto Rondón y Cravo Norte. La aparición de sembradíos de coca en Tame hacia 1999 fue uno de los factores para que las organizaciones de autodefensa decidieran incursionar con el fin de obtener ganancias provenientes del narcotráfico. La estrategia de penetración se consolidó en municipios con baja presencia guerrillera como Cravo Norte y Puerto Rondón y luego se proyectó hacia la zona cocalera de Tame y la cabecera de Arauca. En el 2005, las autodefensas se desmovilizaron del departamento.

La coca (Erythroxylum coca) y su cultivo, introducidos al departamento de Arauca a finales del decenio del noventa, articularon a la región los actores de violencia anteriormente descritos, paramilitarismo y guerrilla, generando una dinámica de conflicto muy cerrada entre los actores armados, el movimiento social y la población campesina. Hablar de la coca como elemento modelador de la historia económica de estas regiones puede ser apresurado, sin embargo ha sido un 
elemento clave de la realidad contemporánea y en definitiva, un cultivo de gran impacto que ha causado grandes costos en el campo económico, social y ambiental. Arauca se convierte definitivamente en un departamento cocalero porque se evidencia la siembra ilegal de coca y adicionalmente se constata el procesamiento y el tráfico de su derivado, la cocaína, siendo en este sentido Tame el municipio dominante. Según resultados de los censos realizados por la Oficina contra la Droga y el Delito de las Naciones Unidas (2008), en el departamento de Arauca los cultivos de coca fueron detectados por primera vez en el año 2000 , en un área aproximada de 1.000 ha y aumentaron a más de 2.000 ha entre 2001 y 2002. En 2003, se mantuvo la superficie cultivada en 2.000 ha, se asperjaron para su destrucción 12.000 ha y el cultivo de coca disminuyó en 500 ha, a diciembre del mismo año. Sin embargo, se incrementó a 2.695 ha en el 2007, a pesar de la erradicación manual adicional de 660 ha. Por los datos reportados para Arauca se deduce que existe una gran rotación de los cultivos de coca dentro del departamento, es así que se encuentran 799 ha de nuevos cultivos y 6.765 ha abandonadas, aunque también se reporta una rotación bastante baja, de 310 ha temporalmente estable, para un total de 7.874 ha en una zona de influencia de 335.552 ha. El área promedio de los lotes sembrados con coca se redujo a menos de la mitad, pasando de 2.46 ha en el año 2001 a 1.17 ha en el año 2006. El índice de estabilidad es del $4.0 \%$. Para este periodo las áreas sembradas con coca en el departamento de Arauca representaban el 2,70\% del total nacional, con una tendencia expansiva hacia el noroccidente del departamento, en la región conocida como piedemonte araucano (ONU, 2008).

La aparición del cultivo de coca en algunos espacios rurales del departamento de Arauca trajo como lógica consecuencia un cambio en el uso de sus suelos en estos espacios, tradicionalmente de vocación ganadera extensiva, desplazando a otros cultivos de la zona, o interrumpiendo las practicas de descanso para la recuperación natural de su fertilidad. Las características y condiciones del cultivo de coca que requirió la intensificación del uso del suelo para el cultivo propiamente dicho y la gran intervención y presencia humana para las cosechas, impactaron negativamente estas áreas y sus entornos vecinos, incrementando el deterioro ambiental. Así, las coberturas boscosas, como bosques primario y secundario y rastrojo alto, al igual que otras coberturas vegetales como pastos, cultivos lícitos y otras, fueron las más afectadas (reemplazadas) con la introducción del cultivo de coca durante el periodo 2001-2006 (Tabla 4), teniendo sus mayores picos en los periodos 20012002 y 2004-2005. Arauca ocupaba en este periodo el décimo lugar en afectación de estas coberturas destinadas al cultivo de coca, representando, en términos de área cultivada, el $3 \%$ del total nacional (Naciones Unidas, 2008). 
Tabla 4. Coberturas vegetales reemplazadas por coca (Erythroxylum coca), 2001-2006

\begin{tabular}{|c|c|c|c|c|c|c|}
\hline \multicolumn{7}{|c|}{ Vegetación reemplazada por coca (Erythroxylum coca) (has) } \\
\hline $\begin{array}{c}\text { Bosque } \\
\text { Primario }\end{array}$ & $\begin{array}{c}\text { Bosque } \\
\text { Secundario }\end{array}$ & Rastrojo Alto & Subtotal & $\begin{array}{c}\text { Otras coberturas vegetales } \\
\text { cultivos } \\
\text { cúcitos, otros }\end{array}$ & Subtotal & Total \\
\hline 553 & 1.598 & 183 & 2.334 & 3.284 & 3.284 & 5.618 \\
\hline
\end{tabular}

Fuente: Naciones Unidas (2008). Análisis multitemporal de cultivos de coca período 2001-2006.

\section{El petróleo como agente histórico de integración de Arauca en la dinámica colombiana}

Hacia 1904 se iniciaron las primeras exploraciones petroleras en el territorio colombiano, que luego tendrían un significativo efecto transformador en diferentes partes del departamento de Arauca. Uno de los primeros frentes de exploración fue el Magdalena Medio, en cercanías de Barrancabermeja, cuyos yacimientos conocían y explotaban los indígenas Yariguí desde antes de la llegada de los españoles, usándolos para calafetear embarcaciones (Avellaneda, 1998), allí se estableció la Concesión de Mares. Otro fue el territorio Barí o Motilón en el norte de Santander, cerca de la frontera con Venezuela, cuyas poblaciones sufrieron un duro impacto, allí se otorgó la Concesión Barco. Ambas regiones corresponden a áreas selváticas que desde entonces empezarían a ser transformadas. Colombia hizo su primera exportación por el oleoducto y puerto de Mamonal, departamento de Bolívar, en 1926. También se presentó alguna exploración en la costa Caribe, en particular en Repelón, Usiacurí, Baranoa (departamento de Atlántico), Turbaco (departamento de Bolívar), y San Andrés de Sotavento (departamento de Córdoba). El resultado de las exploraciones después de 1920 se hizo sentir con fuerza por la importancia de los derivados del petróleo en todos los renglones de la industria moderna. Efectivamente, se hizo materia prima indispensable en los campos de la química (medicina, plásticos, etc.), de los combustibles (gasolina, gas, diesel, etc.), del transporte (neumáticos, partes automotores, etc.), vías de comunicación (asfaltos y pavimentos), de la agroindustria (abonos, fungicidas, insumos) y muchos más renglones. Los efectos de la industria de los hidrocarburos se empezaron a sentir a finales del siglo XX cuando la estabilidad de la atmósfera y de las fuentes hídricas se alteró por las emanaciones contaminantes producida por la suma de dichas industrias y sus productos.

En el departamento de Arauca, en particular, se hicieron los primeros descubrimientos petrolíferos importantes en la década de los setenta del siglo XX, desempeñando un rol protagónico en la reestructuración del espacio geográfico del departamento, en cuanto lo articuló e integró al espacio regional y nacional, 
puesto que en casi todos los ámbitos de acción gubernamental hasta entonces permanecía marginado por parte del Estado. Igualmente, le generó nuevas actividades y dinámicas espaciales que marcaron su posterior evolución con incidencia en una reconfiguración de su espacio geográfico.

\section{El petróleo en la modificación del espacio araucano}

El contrato de explotación de petróleo por la modalidad de concesión ofrecía grandes ventajas para el capital extranjero y fue la que predominó en Colombia desde 1905. A partir del decenio del 40 del siglo XX, compañías multinacionales comenzaron la búsqueda de petróleo en la Orinoquia colombiana; hacia los años 70 los expertos mostraron las bondades petrolíferas del subsuelo en la región y se encontraron los primeros hallazgos en los municipios de Trinidad y Tocaría, Apiay y Cubarral (Casanare y Meta respectivamente). La explotación de los recursos petroleros en el departamento de Arauca se inició en 1959 con la perforación del pozo la Heliera 1 (Puerto Rondón) y el de Tame 1 por la Socony-Mobil y en 1960 por la Shell. En 1980, Intercol perfora los pozos Arauca 1 y 2 en Saravena, y en 1981, ECOPETROL perfora el pozo Río Ele. En 1982, la Occidental, como parte de la asociación Cravo Norte, perfora el Pozo No. 1 que posibilita el hallazgo del campo Caño Limón, en septiembre de 1983. Entonces, el departamento de Arauca registraba reservas en sus yacimientos de 1,1 billones de barriles (Ecopetrol S.A., 2008), localizados en el municipio de Arauca. Un año después se descubrieron otros pozos como la Yuca y Matanegra, hallazgos que marcaron el repunte de la producción de crudo a nivel nacional, alcanzando su autosuficiencia petrolera a partir de 1986 y su condición de exportador neto de crudo (Aguilar et al., 1998).

La importancia de los yacimientos de petróleo en Arauca con proyección significativa en la economía colombiana de exportación, son hechos concretos de transformaciones profundas en el desarrollo histórico de la estructura del espacio departamental, toda vez que propiciaron modificaciones notorias, no solo en el departamento, sino en su región, la Orinoquia y en el país. Los descubrimientos hidrocarburíferos abrieron las posibilidades de desarrollo de una región marginada, como era el departamento de Arauca y su contexto geográfico, la Orinoquia colombiana, especialmente a partir del hallazgo y explotación del actual campo Caño Limón (1983). Las estructuras territoriales cambiaron notablemente y es el petróleo el que ingresa a desempeñar desde entonces un rol integrador fundamental entre los tres niveles espaciales: departamental, regional y nacional.

En los años noventa, Colombia prolongó su autosuficiencia petrolera con el descubrimiento de los yacimientos gigantes Cusiana y Cupiagua en el departamento de Casanare (Figura 4) en el piedemonte llanero de la región orinoquense, recurso explotado en asocio con la British Petroleum Company BP. Este acontecimiento transcendental para la economía de Co- 
lombia y en consecuencia con dividendos positivos -en términos económicos- para Arauca y la Orinoquia, ocasionó una intensa dinámica que transformó el curso del desarrollo del espacio araucano y se convierte por lo tanto en un hecho geohistórico al reconfigurar económica, social, cultural y geográficamente al departamento.

En primera instancia, el recurso hidrocarburífero, como elemento de la reconfiguración del espacio araucano, se manifiesta en la implementación de infraestructuras para el transporte de crudo, hasta entonces extrañas en el departamento, cuyo oleoducto se extiende desde el mega-campo Caño Limón hasta Coveñas (Sucre), puerto sobre el mar Caribe. El trayecto del oleoducto cubre los municipios de Arauca, Arauquita y Saravena (Arauca), Cubará (Boyacá), Toledo, Chinácota, San Cayetano, el
Zulia, Tibú, el Tarra, Convención, el Carmen (Norte de Santander), Aguachica (Cesar), el Banco, Plato (Magdalena), Magangué, (Bolívar) y Sincelejo (Sucre), como se puede advertir en la Figura 5.

Además fue necesaria la construcción de estaciones de bombeo y almacenamientos, de vías que enlazaran los diferentes sitios asociados con la actividad y de puntos de servicios sociales como centros de salud, educación, recreación y alojamientos de la población involucrada en dicha actividad económica. Grandes extensiones de territorio, antes dedicadas a la agricultura y ganadería extensiva, debieron cambiar su uso para pertenecer al ámbito de la explotación del petróleo y actividades colaterales. Los resultados de toda la anterior dinámica, se traducen en profundas modificaciones de la estructura del espacio geográfico araucano.

Figura 4. Principales descubrimientos petrolíferos en Colombia.

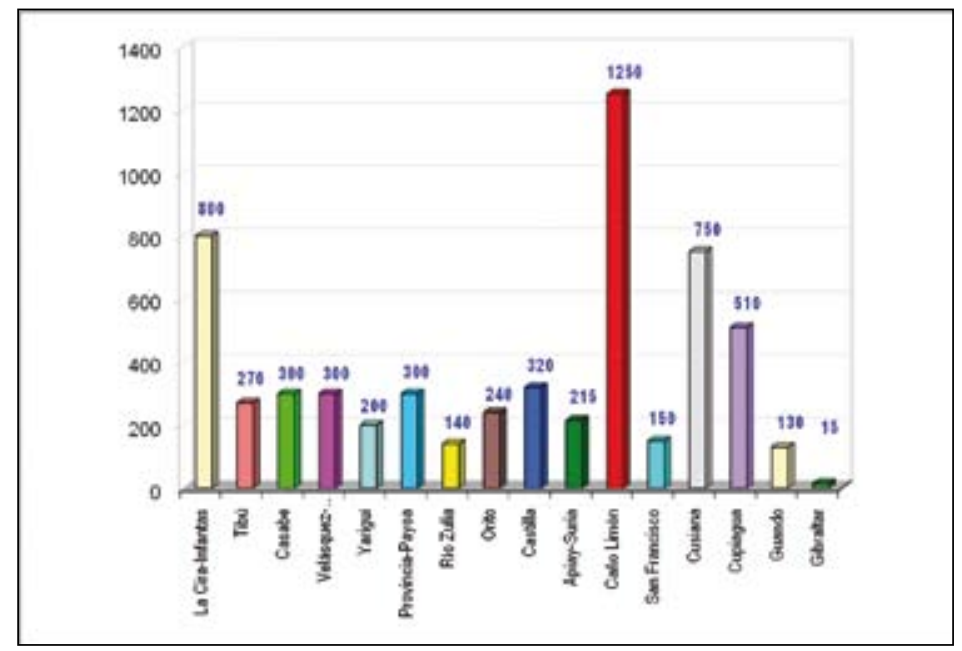

Fuente: Ecopetrol S.A., 2008. 
Figura 5. Infraestructura petrolera en Colombia, 2005.

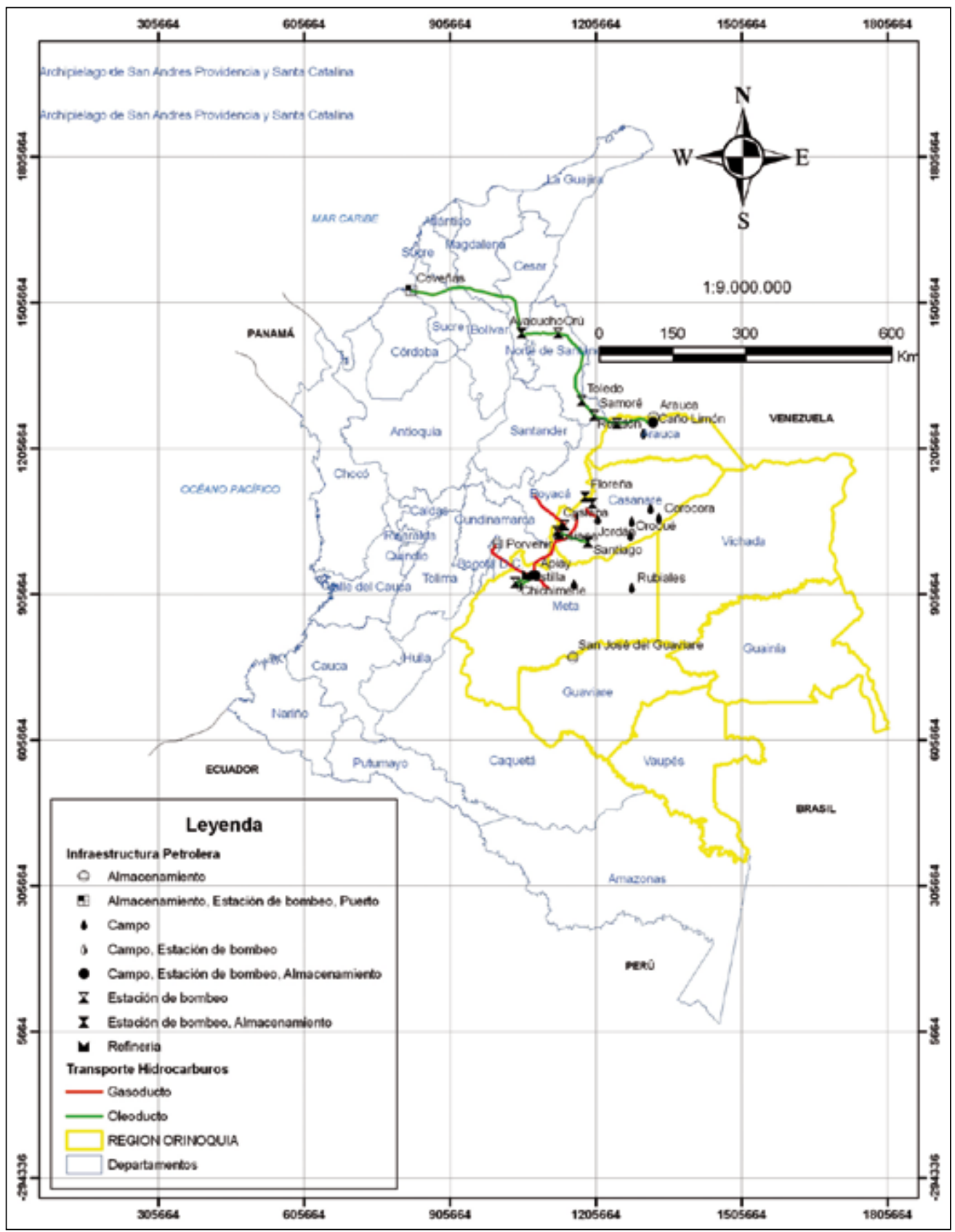

Fuente: Ecopetrol S.A., 2008. 


\section{Conclusiones}

Luego del análisis de los resultados obtenidos, se concluye que:

1. A partir de 1950 , el territorio del actual departamento de Arauca configura cambios notables en la estructura de la ocupación del espacio geográfico y distribución de la población, resultado de factores dominantes que se gestan desde 1930 y que particularizan el espacio local y su contexto regional. Estos se identifican, inicialmente, en la violencia entre los partidos políticos liberal y conservador desarrollada hacia mediados de siglo XX, la que generó importantes desplazamientos de población hacia cabeceras municipales, capitales de departamentos y Bogotá, el centro de la República de Colombia, así como el estancamiento de las actividades productivas y disminución de la población por el derramamiento de sangre durante dicho fenómeno coyuntural y posteriormente, con el descubrimiento del campo Caño Limón en 1983. Su importancia como hito histórico es transcendental para Arauca, puesto que da paso a la intensificación de la actividad económica que se extiende e involucra a la población, la cultura y el espacio dentro de nuevos esquemas de organización, en el que se configuran las actuales estructuras socioespaciales de áreas urbanas y rurales del departamento de Arauca y de la región de la Orinoquia. La densificación de la población se promueve por el conjunto de dinámicas de diverso tipo que desarrolla la actividad de exploración y explotación de este hidrocarburo, que se transporta a través del oleoducto Caño Limón hasta Coveñas, puerto de exportación sobre el mar Caribe.

La estructura poblacional departamental presenta dos sub periodos notoriamente diferenciados en su composición poblacional, el primero, entre 1951 y 1985, y el segundo, entre 1985 y 2005 . Entre 1951 y 1985 el crecimiento de su población es gradual (1.672 habitantes/año durante 34 años) predominando como factores la movilidad interna, violencia política y la aceleración tecnológica en la producción generada por presiones externas en la imposición de un modelo de crecimiento económico basado en la sobreexplotación de los recursos naturales y en el desplazamiento de la mano de obra. Entre 1985 y 2005 se registra un acelerado crecimiento (8.102 habitantes/ año durante 20 años) concentrado en el municipio de Arauca, cuya causa principal está en el descubrimiento del mega campo de Caño Limón en 1983, que atrae población en torno a su explotación.

2. La concentración de tierras en el departamento de Arauca fue y es una forma de dominio económico y político del territorio, particularmente en los espacios sabaneros, más que en los de piedemonte y montañosos. Los primeros procesos colonizadores sobre tierras vírgenes del país, así como la presencia de actores armados 
ilegales y la explotación de recursos petroleros por las transnacionales patrocinadas por el Estado, son las causas de esta concentración y del desplazamiento de campesinos con propiedades de tierra medianas y pequeñas, como se pudo advertir particularmente para el año 1985. Esta estructura típica de concentración de tierras genera conflictos de uso, priorizando la ganadería extensiva en detrimento de otros sistemas productivos que podrían ser más rentables económica y socialmente (agricultura intensiva de productos adaptados a la zona como hortalizas, maíz, sorgo y arroz) para un número mayor de habitantes de la zona (campesinos e indígenas) y para venta en el resto de Colombia y exportación a otros países, dentro de la dinámica mundial de la globalización.

3. La penetración a partir de los años sesenta de grupos armados al margen de la ley como el Ejército de Liberación Nacional (ELN), Fuerzas Armadas Revolucionarias de Colombia (FARC) y las Autodefensas Unidas de Colombia (AUC), incide igualmente en la reconfiguración del espacio araucano mediante el dominio de facto impuesto en las áreas municipales, cambiando las actividades productivas, la tenencia de la tierra y los movimientos de población. El campo petrolero de Caño Limón y su infraestructura fueron utilizados por los grupos armados ilegales como elementos de coerción contra el Estado, haciendo que sus acciones cobraran mayor valor y poder sobre la población araucana y el manejo del espacio, presentándose como otro factor estructurante territorial.

4. Por último, el petróleo, recurso natural cuyo campo más importante a nivel nacional se encuentra en el departamento de Arauca, se constituye desde 1983 y hasta el presente, en el estructurante más influyente de los espacios rurales y urbanos del departamento, por la afectación en todas las dimensiones de la sociedad, resaltó al departamento en el contexto nacional y suprimió la lejanía que lo aislaba del país y la marginalidad en las dinámicas económicas. La construcción de infraestructuras de explotación, almacenamiento y transporte del recurso, es el elemento tangible de la reconfiguración del espacio araucano. Resumiendo, a partir del descubrimiento de la riqueza petrolera de Caño Limón se instauran nuevas dinámicas socioeconómicas que transforman la estructura territorial de un espacio fraccionado y en condición de marginalidad de la acción estatal. Novedosas actividades económicas aparecen en el departamento de Arauca que inducen cambios significativos en las estructuras de la población, en la ocupación y el manejo del espacio. Nuevos actores sociales participan en la dinámica productiva en sus diferentes niveles y variables, pero también intervienen los grupos al margen de la ley atraídos por los beneficios económicos resultantes de la explotación petrolera. 


\section{Literatura citada}

Acción Social. (2002, 2003, 2004, 2005). Estadísticas sobre desplazamiento forzado, personas expulsadas personas recibidas. Obtenido en marzo de 2010 desde http://sigotn.igac.gov.co/sigotn/2011

Aguilar, O., Galeano, C. \& Pérez, L. (1998). Petróleo y desarrollo. En Fajardo, D. (Ed.). Colombia Orinoco. Bogotá: Fondo FEN.

Avellaneda, A. (1998). Petróleo, colonización y medio ambiente en Colombia: de la Tora a Cusiana. Bogotá: ECOE.

Avellaneda, A. (2004). Petróleo, ambiente y conflicto en Colombia. En Cárdenas, M. y

Rodríguez, M. (Ed.). Guerra, sociedad y medio ambiente. Bogotá: Fondo Nacional Ambiental

Braudel, F. (1950). Les responsabilités de I'histoire. Cahiers internationaux de sociologie. Paris: Paris X.

Centro de Investigaciones para el Desarrollo. (2005). Plan estratégico de productividad y competitividad del departamento de Arauca. Bogotá: Convenio Gobernación de Arauca-Universidad Nacional de Colombia.

CORPOICA. (1997). Evaluación biofísica de tierras del departamento de Arauca con fines agropecuarios. Bogotá.

Departamento Administrativo Nacional de Estadística. DANE. (2005). Censos 1951 al 2005. Bogotá.

Dollfus, O. (1976). El espacio geográfico. Colección ¿qué sé? Barcelona: Oikos Tau.

Ecopetrol. (2008). Historia. Perspectiva histórica. Recuperado en julio, 2008, de http://www.ecopetrol.com. co/contenido.aspx? catID $=32 \&$ conID $=36271$

Gobernación de Arauca. (2006). Sistema de información geográfica departamento de Arauca. Arauca, Colombia.

Grataloup, Ch. (1996). Lieux D'histoire. Essaai de Géohistoire Systématique. Montpellier: GIP-RECLUS.

Hurtado, O. (1994). Geohistoria de Villa de Cura y su área de influencia. Caracas: Asociación de Educadores de América Latina y del Caribe AELAC.

Instituto Geográfico Agustín Codazzi IGAC. (2005). Estadística catastral de predios rurales del departamento de Arauca. Colombia. Entregado por la subdirección de Catastro del IGAC, 2008.

Márquez, G. (2001). Transformación de ecosistemas colombianos. Departamento de Arauca. Bogotá: Biología, Facultad de Ciencias e Instituto de Estudios Ambientales.

Organización de las Naciones Unidas ONU. (2008). Colombia monitoreo de cultivos de coca. Colombia: Oficina contra la Droga y el Delito.

Red de Solidaridad Social. (2005). Estadísticas desplazamiento forzado. (Información solicitada y entregada oficialmente por esta entidad al IGAC).

Santos, M. (1996). Metamorfosis del espacio habitado. Barcelona: Oikus-Tau.

Santos, M. (2000). La naturaleza del espacio: técnica y tiempo. Razón y emoción. Barcelona, España: Ariel.

Tovar, R. (1996). El enfoque geohistórico. (2 Ed.). Valencia: Universidad Carabobo.

Vicepresidencia de la República. (2003). Los derechos humanos en el departamento de Arauca. Programa presidencial de derechos humanos y derecho internacional humanitario. Obtenido en marzo de 2010, desde http://www.derechoshumanos.gov.co/Pna/documents/2010/arauca/arauca.pdf

Recepción: 14 de abril de 2011

Evaluación: 25 de mayo de 2011

Aprobación: 23 de junio de 2011

172 Betty Esther Mendoza Padilla 